Bull. Austral. Math. Soc.

$46 \mathrm{~B} 20,46 \mathrm{~B} 03$

VOL. 52 (1995) [225-229]

\title{
REMARKS ON SYMMETRIC SMOOTH NORMS
}

\author{
P. HÁJEK AND V. Zizler
}

A characterisation is given of separable Banach spaces with symmetric Schauder bases which admit equivalent symmetric norms that are Gâteaux differentiable or uniformly rotund in every direction. Some applications to questions on distortion of norms on $\ell_{\infty}$ are discussed.

In his paper [6], Troyanski proved that a nonseparable Banach space $X$ with a symmetric Schauder basis admits an equivalent uniformly Gâteaux differentiable norm if and only if $X$ is not isomorphic to a space $\ell_{1}(\Gamma)$. He also showed in [6] a dual result on norms that are uniformly rotund in every direction. In [3] a canonical extension of symmetric norms on separable Banach spaces to their nonseparable copies was considered. This way a nonseparable reflexive space $T$ with a symmetric norm was introduced with the property that no nonseparable subspace of $T$ linearly injects into any superreflexive Banach space. Note that by Troyanski's results mentioned above the space $T$ admits an equivalent norm that is uniformly Gâteaux differentiable and uniformly rotund in every direction. It follows from the results of Argyros and Farmaki [1, Theorem 2.5] that the unit ball of $T$ in its weak topology is a uniform Eberlein compact, that is, homeomorphic to a subset of a Hilbert space in its weak topology. The purpose of this note is to show that by combining the ideas in [6] and [3], a characterisation can be obtained of separable spaces which admit symmetric norms that are Gâteaux differentiable or uniformly rotund in every direction. We let $X$ be a separable Banach space with a symmetric Schauder basis $\left\{e_{i}\right\}_{i \in N}$ and denote by II (respectively, $\Theta$ ) the set of all permutations of $\mathbb{N}$ (respectively, injections of $\mathbb{N}$ into $\mathbb{N}$ ). Recall that an equivalent norm $\|\cdot\| \|$ on such $X$ is called symmetric if $\|x\|=\|\| x_{\pi} \|$ for every $x \in X$ and $\pi \in \Pi$ where $x_{\pi}=\sum_{i \in \mathbf{N}} x_{i} e_{\pi(i)}$. Similarly we can define $x_{\theta}=\sum_{i \in \mathbf{N}} x_{i} e_{\theta(i)} \in X$ for $\theta \in \Theta$ and note that $\left\|x_{\theta}\right\|=\|x\|$ for every $x \in X$. To see this, we first use the unconditionality of the basis $\left\{e_{i}\right\}_{i \in N}$ to observe that for every $\varepsilon>0$ there is $n_{0} \in \mathbb{N}$ such that $\left\|\mid \sum_{i \in A} x_{i} e_{i}\right\|<\varepsilon$ for every finite set $A \subset \mathbb{N}$ with min $A \geqslant n_{0}$. From the symmetry of the norm $\|\cdot\| \|$ we have that there is $m_{0} \in \mathbb{N}$ such that $\left\|\sum_{\theta(i) \in B} x_{i} e_{\theta(i)}\right\|<\varepsilon$

Received 14th November, 1994

Supported in part by NSERC (Canada)

Copyright Clearance Centre, Inc. Serial-fee code: 0004-9729/95 \$A2.00+0.00. 
whenever $B$ is a finite set in $\mathbb{N}$ with $\min B \geqslant m_{0}$. Therefore $\sum_{i \in \mathbb{N}} x_{i} e_{\theta(i)}$ is a convergent series and we can see that $\|x\|=\left\|x_{\theta}\right\|$.

Standard notation is used in this note. In particular, we denote by $c_{0}$ respectively, $\ell_{p}$ or $\ell_{\infty}$ the spaces $c_{0}(\mathbb{N})$ respectively, $\ell_{p}(\mathbb{N})$ or $\ell_{\infty}(\mathbb{N})$. We refer to [5] and [2] for some unexplained notion and results.

We now define a canonical nonseparable extension of symmetric norms.

DEFINITION 1: Let $X$ be a separable Banach space with a symmetric Schauder basis $\left\{e_{i}\right\}_{i \in \mathrm{N}}$ and a symmetric norm $\|\cdot\|_{X}$. Let $\Gamma$ be an uncountable ordinal and $X(\Gamma)$ be the vector space of all countably supported vectors on $\Gamma$ with the norm $\|\cdot\|_{\Gamma}$ defined as follows:

If, formally, $x=\sum_{\lambda \in \Gamma} x_{\lambda} e_{\lambda}$ where $e_{\lambda}$ are the unit vectors on $\Gamma$, choose a vector $y \in X, \quad y=\sum_{i \in \mathbb{N}} y_{i} e_{i}$ and an injection $I$ of $\mathbb{N}$ into $\Gamma$ such that $x_{\lambda}=y_{i}$ for $\lambda=I(i)$ and $x_{\lambda}=0$ otherwise. Then put $\|x\|_{\Gamma}=\|y\|_{X}$.

The space $\left(X(\Gamma),\|\cdot\|_{\Gamma}\right)$ is a nonseparable Banach space with the symmetric basis $\left\{e_{\lambda}\right\}_{\lambda \in \Gamma}$ and the norm $\|\cdot\|_{\Gamma}$ is a symmetric norm on $X(\Gamma)$.

The correctness of Definition 1 follows from the fact on the norms of the elements $x_{\theta}$ discussed above. To see this, given a sequence $\left\{x^{k}\right\}_{k \in \mathrm{N}}$ of vectors in $X(\Gamma)$, note that $\operatorname{card}\left(\bigcup_{k \in \mathbb{N}} \operatorname{supp}\left(x^{k}\right)\right) \leqslant \aleph_{0}$ and we can define an injection $I$ of $\mathbb{N}$ into $\Gamma$ and a sequence $\left\{y^{k}\right\}_{k \in \mathrm{N}}$ of vectors in $X$ such that $x_{\lambda}^{k}=y_{i}^{k}$ for $\lambda=I(i)$ and $x_{\lambda}^{k}=0$ otherwise. We can see that $\|\cdot\|_{\Gamma}$ is indeed a norm since it is homogeneous and convex, as $\left\|x^{1}+x^{2}\right\|_{\Gamma}=\left\|y^{1}+y^{2}\right\|_{X} \leqslant\left\|y^{1}\right\|_{X}+\left\|y^{2}\right\|_{X}=\left\|x^{1}\right\|_{\Gamma}+\left\|x^{2}\right\|_{\Gamma}$. The completeness of the norm $\|\cdot\|_{\Gamma}$ can be seen similarly: If $\left\{x^{k}\right\}_{k \in N}$ is a Cauchy sequence in $\left(X(\Gamma),\|\cdot\|_{\Gamma}\right)$ and we define an injection $I$ and a sequence $\left\{y^{k}\right\}_{k \in \mathbb{N}}$ as above, then $\left\{y^{k}\right\}_{k \in \mathbb{N}}$ is a Cauchy sequence in $X$. Thus $\lim _{k} y^{k}=y$ for some $y \in X$. Then $\lim x^{k}=x$ in $X(\Gamma)$, where $x_{\lambda}=y_{i}$ for $\lambda=I(i)$ and $x_{\lambda}=0$ otherwise. By using the idea of passing from a sequence $\left\{x^{k}\right\}_{k \in \mathrm{N}}$ in $X(\Gamma)$ to its copy $\left\{y^{k}\right\}$ in $X$ we can see that the usual properties of smoothness or rotundity of the norm $\|\cdot\|$ on $X$ are carried over to the norm $\|\cdot\|_{\Gamma}$, as any such property can be expressed by using sequences of vectors in $X(\Gamma)$.

We can characterise spaces with symmetric bases that are isomorphic to $c_{0}$ or $\ell_{1}$ by the following statement.

Proposition 2. Let $X$ be a separable Banach space with a symmetric Schauder basis. Then the space $X$ admits an equivalent symmetric Gâteaux differentiable norm if and only if $X$ admits an equivalent symmetric uniformly Gâteaux differentiable norm if and only if $X$ is not isomorphic to $\ell_{1}$.

Proof: Let $\left\{e_{i}\right\}_{i \in \mathrm{N}}$ be a symmetric Schauder basis of $X$. If $X$ is not isomorphic 
to $\ell_{1}$ then the basis $\left\{e_{i}\right\}_{i \in \mathbf{N}}$ is not equivalent to the standard unit vector basis in $\ell_{1}$ and from the proof of Theorem 2 in [6] it follows that $X^{*}$ admits an equivalent dual norm $\|\cdot\|^{*}$ that is $w^{*}$-uniformly rotund and symmetric on the norm closed linear span of the biorthogonal functionals to $\left\{e_{i}\right\}_{i \in \mathbb{N}}$. Therefore its predual norm $\|\cdot\|$ on $X$ is symmetric in $\left\{e_{i}\right\}_{i \in N}$ and uniformly Gâteaux differentiable by Šmulyan's duality result (see for example, [2, Theorem II.6.7]). On the other hand, let $\|\cdot\|_{X}$ be an equivalent symmetric and Gâteaux differentiable norm on $X, T$ be an isomorphism of $X$ onto $\ell_{1}$ and $u_{i}$ be the standard unit vectors in $\ell_{1}$. Define an equivalent norm $\|\cdot\|$ on $\ell_{1}$ for $y=\sum_{i \in \mathbf{N}} \lambda_{i} u_{i}$ by $\|y\|=\left\|\sum_{i \in \mathbb{N}} \lambda_{i} e_{i}\right\|_{X}$. To see that this is a good definition we take into account that the basis $\left\{T e_{i}\right\}_{i \in \mathbb{N}}$ is equivalent to the standard unit vector basis of $\ell_{1}$ as any normalised unconditional basis is (see for example, [5, Proposition 2.6.9]). The norm $\|\cdot \mid\|$ is symmetric on $\ell_{1}$ with respect to $\left\{u_{i}\right\}_{i \in \mathbb{N}}$. Therefore a nonseparable extension $\left(\ell_{1}(\Gamma),\|\cdot\|_{\Gamma}\right)$, which is an equivalent renorming of the usual space $\ell_{1}(\Gamma)$, admits an equivalent Gâteaux differentiable norm. This is impossible by Day's result (see for example, [2, p. 59]).

Propos I I I IN 3. Let $X$ be a separable Banach space with a symmetric Schauder basis. Then $X$ admits an equivalent symmetric Fréchet differentiable norm if and only if $X$ does not contain an isomorphic copy of $\ell_{1}$.

Proof: Let $\left\{e_{i}\right\}_{i \in \mathbf{N}}$ be a symmetric Schauder basis of $X$. If $X$ does not contain an isomorphic copy of $\ell_{1}$, then by James' theorem, the basis $\left\{e_{i}\right\}_{i \in \mathbf{N}}$ is shrinking (see for example, [5, Theorem 1.c.9]). Therefore the biorthogonal functionals to the basis $\left\{e_{i}\right\}_{i \in \mathbf{N}}$ form a symmetric basis of $X^{*}$. Since Troyanski's classical construction in [8] produces an equivalent dual norm on $X^{*}$ that is symmetric and locally uniformly rotund (see for example, [2, Lemma VII.1.7]), by using the Šmulyan duality result we obtain an equivalent symmetric Fréchet differentiable norm on $X$ (see for example, $[2$, Proposition II.1.5]). On the other hand, if a separable $X$ admits an equivalent Fréchet differentiable norm, then $X^{*}$ is separable (see for example, [2, Corollary II.3.3]) and thus $X$ cannot contain an isomorphic copy of $\ell_{1}$.

It is a result of Day, James and Swaminathan that the space $c_{0}(\Gamma)$ admits no equivalent norm which is uniformly rotund in every direction if $\Gamma$ is uncountable (see for example, [2, Proposition II.7.9]). Using this result and the proof of Theorem 1 in [6], similarly as in the proof of Proposition 2 above, we obtain the following result.

PRoposition 4. Let $X$ be a separable Banach space with a symmetric Schauder basis. Then $X$ admits an equivalent symmetric norm that is uniformly rotund in every direction if and only if $X$ is not isomorphic to $c_{0}$.

Note that it follows from the proof of Theorem V.1.5 in [2] that $c_{0}$ admits an equivalent symmetric rotund norm that is a limit, uniform on bounded sets, of sym- 
metric $C^{\infty}$-smooth norms. On the other hand, if $\Gamma$ is uncountable, then $c_{0}(\Gamma)$ admits no rotund $C^{2}$-smooth norm [4]. Hence by the method of a nonseparable extension we obtain that $c_{0}$ admits no equivalent symmetric rotund $C^{2}$-smooth norm. Note that if $\||\cdot|\|$ denotes an equivalent $C^{\infty}$-smooth norm on $c_{0}$, then the norm $\|\cdot\|$ defined on $c_{0}$ by $\|x\|=\|x\|+\left(\sum\left(1 / 2^{i}\right) x_{i}^{2}\right)^{1 / 2}$ is an equivalent rotund $C^{\infty}$-smooth norm. In [7] Troyanski proved that if $p$ is an odd number, then the space $\ell_{p}(\Gamma)$ admits an equivalent norm that is $p$-times Gâteaux differentiable if and only if $\Gamma$ is countable. Therefore, by using the method of a nonseparable extension, we obtain that $\ell_{p}$ admits no equivalent symmetric $p$-times Gateaux differentiable norm if $p$ is an odd number. If $X$ is a separable Banach space with a symmetric Schauder basis and $X^{*}$ is nonseparable, then there are $\varepsilon>0$ and a subset $A$ of the dual unit ball $B_{X^{*}}$ of $X^{*}$ such that all the $w^{*}$-slices of $A$ are of diameter $\geqslant \varepsilon$ (see for example, [2, Theorem I.5.3]). For $\pi \in \Pi$, let $\pi^{*}$ denote the dual operator to the permutation $\pi$-operator on $X$. Then the set $B_{X^{*}}+\overline{\mathrm{conv}^{*}} \omega^{*}\left(\bigcup_{\pi \in \Pi} \pi^{*}(A \cup(-A))\right)$ is the dual unit ball of a symmetric rough norm on $X$ (see for example, [2, Proposition I.1.11]).

We shall say that an equivalent norm $\|\cdot\|$ on $\ell_{\infty}(\Gamma)$ is symmetric if $\left\|x_{\pi}\right\|=\|x\|$ for every $x \in \ell_{\infty}(\Gamma)$ where $x_{\pi}(i)=x_{\pi(i)}$ and $\pi$ is a permutation of $\mathbb{N}$.

With this definition we obtain the following result on distortion of norms on $\ell_{\infty}$.

Proposition 5. Let $\|\cdot\|_{\infty}$ denote the standard sup-norm on $\ell_{\infty}$ and let $\|\cdot\|$ be an equivalent symmetric norm on $\ell_{\infty}$. Then there is a linear isometry of $\left(\ell_{\infty},\|\cdot\|_{\infty}\right)$ into $\left(\ell_{\infty},\|\cdot\|\right)$.

Proof: Let an operator $T$ of $\ell_{\infty}$ be defined by $T\left(\sum_{i=1}^{\infty} \alpha_{i} e_{i}\right)=\sum_{i=1}^{\infty} \alpha_{i} e_{2 i}$, where we consider the formal sums of the unit vectors $\left\{e_{i}\right\}$ in $\ell_{\infty}$. Define a norm $\|\cdot\|_{T}$ on $\ell_{\infty}$ by $\|x\|_{T}=\|T x\|$ for $x \in \ell_{\infty}$. The norm \|\|$_{T}$ is an equivalent symmetric norm on $\ell_{\infty}$ and it has the property that $\|x\|_{T}=\|y\|_{T}$ whenever $y$ is obtained from $x$ by adding some zero coordinates. If $\Gamma$ is an uncountable ordinal and $\ell_{\infty}^{c}(\Gamma)$ denotes the subspace of $\ell_{\infty}(\Gamma)$ formed by countably supported vectors, similarly as in Definition 1 we can define a norm $\|\cdot\|_{\Gamma}$ on $\ell_{\infty}^{c}(\Gamma)$ by $\|x\|_{\Gamma}=\|\tilde{x}\|_{T}$, where $\tilde{x} \in \ell_{\infty}$ and $x \in \ell_{\infty}^{c}(\Gamma)$ have the same structure of their coordinates. By Partington's result (see for example [2, Theorem II.7.12]), for every equivalent norm on $\ell_{\infty}^{c}(\Gamma)$ and in particular for the norm $\|\cdot\|_{\Gamma}$, the space $\left(\ell_{\infty}^{c}(\Gamma),\|\cdot\|_{\Gamma}\right)$ contains an isometric copy of $\left(\ell_{\infty},\|\cdot\|_{\infty}\right)$ such that its total support on $\Gamma$ is countable. We can now isometrically transfer this copy back to $\left(\ell_{\infty},\|\cdot\|_{T}\right)$ and note that $T$ is an isometry of $\left(\ell_{\infty},\|\cdot\|_{T}\right)$ onto the subspace of $\ell_{\infty}$ formed by vectors whose odd coordinates are zero, equipped with the norm $\|\cdot\|$.

COROLlary 6 . The space $\ell_{\infty}$ admits no equivalent symmetric rotund norm and admits no continuous (not necessarily equivalent) symmetric Gâteaux differentiable 
norm.

Proof: Since $\left(\ell_{\infty},\|\cdot\|_{\infty}\right)$ is not rotund, the first statement on rotund norms follows from Proposition 4. Alternatively, this part can be shown as follows: Assume that \|\|$\cdot \|$ is an equivalent symmetric rotund norm on $\ell_{\infty}$. Consider $x \in \ell_{\infty}$ such that its coordinates attain an infinite number of times every value from the set $\{0,(1 / 2), 1\}$ and no other values. Choose a permutation $\pi \in \Pi$ that fixes all coordinates but two with values 1 and 0 and these are interchanged. Then $\left(x_{\pi}+x\right) / 2=x_{\rho}$ for some $\rho \in I$. Hence $\|x\|=\left\|x_{\pi}\right\|=\left\|\left(x_{\pi}+x\right) / 2\right\|$, , contradicting the rotundity of $\|\cdot\|$. If $\ell_{\infty}$ admitted a continuous symmetric Gâteaux differentiable norm, similarly as in the proof of Proposition 5 we would produce a continuous Gâteaux differentiable norm on $\ell_{\infty}^{c}(\Gamma)$ for $\Gamma$ uncountable. However, by Haydon's result, the space $\ell_{\infty}^{c}(\Gamma)$ admits no continuous Gâteaux differentiable norm if $\Gamma$ is uncountable (see for example, [2, p. 89]).

Note that the assumption on the symmetry cannot be dropped in the statements of Proposition 5 or Corollary 6 as there are equivalent rotund norms as well as continuous Gâteaux differentiable norms on $\ell_{\infty}$ that are easily produced by linear injections into $\ell_{2}$.

\section{REFERENCES}

[1] S. Argyros and V. Farmaki, 'On the structure of weakly compact subsets of Hilbert spaces and applications to the geometry of Banach spaces', Trans. Amer. Math. Soc. 289 (1985), 409-427.

[2] R. Deville, G. Godefroy and V. Zizler, Smoothness and renormings in Banach spaces, Monographs Surveys Pure Appl. Maths. 64 (Pitman, London, 1993).

[3] P. Hájek, 'Polynomials and injections of Banach spaces into superreflexive spaces', Arch. Math. 63 (1994), 39-44.

[4] P. Hájek, 'On convex functions in $c_{0}\left(w_{1}\right)$ ', Collect. Math. (to appear).

[5] J. Lindenstrauss and L. Tzafriri, Classical Banach spaces I. Sequence spaces (Springer-Verlag, Berlin, Heidelberg, New York, 1977).

[6] S. Troyanski, 'On nonseparable Banach space with a symmetric basis', Studia Math. 53 (1975), 253-263.

[7] S. Troyanski, 'Gâteaux differentiable norms in $L_{p}$ ', Math. Ann. 287 (1990), 221-227.

[8] S. Troyanski, 'Equivalent norms in nonseparable Banach spaces with unconditional basis', Teor. Funktsii Funktsional Anal. i Prilozhen 6 (1968), 59-65. 